\title{
El fin de la protección gonadal en radiografía de pelvis: Una revisión
}

\author{
Pablo Maturana Q. ${ }^{1}$, Marcela Aravena V. ${ }^{1}$, María Ubilla H. ${ }^{1}$, Felipe Allende N $^{\text {*}}$.
}

1. Facultad de Ciencias. Escuela de Tecnología Médica. Universidad Mayor. Santiago, Chile.

The end of gonadal protection in pelvic radiography: A review

\section{Resumen:}

La protección gonadal ha sido durante largo tiempo un importante factor para abordar el requisito "tan bajo como sea razonablemente posible" ALARA. El presente artículo realiza una revisión sistemática sobre la utilidad de la protección gonadal en la radiografía de pelvis en la cual se han destacado los factores que tienen que ver con su realización diaria, es decir, la dosis de radiación involucrada, la radiosensibilidad de las gónadas, la posición del protector gonadal y el factor psicológico de la población en relación con su uso. La incorporación de equipamientos modernos, con dosis y protocolo optimizados, transforma el beneficio de la protección en un tema al menos debatible. Mientras algunos siguen respaldando la medida, otros organismos y autores ya no la respaldan. Es necesario reconsiderar prácticas actuales fundadas en consensos científicos que pueden estar obsoletos y considerar el factor de cambio cultural basados en estos nuevos consensos para su implementación, sin generar mayor preocupación en la población.

Palabras clave: Dosis de radiación; Protección gonadal; Radiografía de pelvis; Radiología pediátrica; Radiosensibilidad.

\begin{abstract}
:
Gonadal protection has long been an important factor in addressing the ALARA "as low as reasonably possible" requirement. This article performs a systematic review on the usefulness of gonadal protection in pelvic radiography, in which the factors that have to do with its daily performance have been highlighted, that is, radiation dose involved, the radiosensitivity of the gonads, the position of the gonadal shielding and the psychological factor of the population in relation to its use. The incorporation of modern equipment, with optimized dose and protocols, transforms the benefit of gonad shielding into an issue that is at least debatable. While some continue to support the measure, other agencies and authors no longer support it. It is necessary to reconsider current practices based on scientific consensus that may be obsolete and consider the factor of cultural change based on these new consensus for its implementation, without generating major concern in the population.
\end{abstract}

Keywords: Gonad shielding; Paediatric radiology; Pelvic x-ray; Radiosensitivity; Radiation dose.

Maturana P., et al. El fin de la protección gonadal en radiografía de pelvis: una revisión. Rev Chil Radiol 2020; 26(2): 40-45.

${ }^{\star}$ Correo electrónico: Felipe Allende / felipe.allende@umayor.cl

Trabajo enviado el 21 de marzo de 2020. Aceptado para publicación el 05 de mayo de 2020. 


\section{Introducción}

En la década de los 50 se registran los primeros trabajos acerca del uso del protector gonadal en la radiografía de pelvis ${ }^{(1,2,3)}$ y hasta el día de hoy sigue siendo un estándar en protección radiológica, esto justificado por la radiosensibilidad de los órganos que están expuestos y por el criterio ALARA, que es uno de los principios fundamentales en la práctica radiológica $^{(4)}$. Los daños producidos por la irradiación de las gónadas podrían ser de tres diferentes tipos: inducción tumoral, efectos hereditarios en los descendientes y deterioro de fertilidad en el individuo irradiado(5). Sin embargo, las gónadas tienen una relativa baja sensibilidad a la inducción de cáncer por irradiación ya que no han sido documentado efectos de manera concluyente. Tampoco, hasta el momento, se ha documentado evidencia directa de que la exposición de los padres a la radiación en bajas dosis (definida por la Comisión Internacional en Protección RadiológicaICRP- a un valor menor o igual a $100 \mathrm{mSv})^{(4)}$ conduzca a un exceso de enfermedad heredable en la progenie. Por último, el deterioro de la fertilidad sí es un efecto documentado y varía en rangos de dosis por sobre 3000 mGy en mujeres y 250 mGy en hombres ${ }^{(5)}$, por ende este sería el efecto con consecuencias más directas. No obstante, hoy en día las dosis que los pacientes reciben en una radiografía de pelvis van de un rango desde los 0.008 a los $0.098 \mathrm{mSv}^{(6)}$, por lo que el riesgo de un posible deterioro en la fertilidad se presenta como casi inexistente.

Tecnólogos médicos y radiólogos han continuado con el legado del paradigma de uso de protección gonadal, sin embargo, la disminución en las dosis de radiación, la implementación de nuevos métodos de control de dosis y los últimos resultados en los estudios sobre los daños asociados a dosis, llevan a pensar en cambiar la mirada del mundo radiológico en torno a la protección gonadal.

El objetivo de este estudio revisar evidencia de los riesgos asociados a la protección gonadal, los que son considerado mayores en comparación a sus beneficios.

\section{Materiales y métodos}

El presente artículo es una revisión sistemática que tiene como objetivo analizar literatura sobre el uso de protector gonadal en la radiografía de pelvis. Se realizó una consulta inicial en NCBI, PubMed, Science Direct entre los años 2000 y 2020 en los idiomas inglés y español. Este estudio adoptó un diseño no experimental.

Para la realización de esta investigación se consideraron sólo exámenes de radiografías de pelvis AP en niños, mujeres y hombres, todos en cualquier rango de edad.

Se excluyeron de este estudio aquellos artículos que describen el uso del protector gonadal en otras técnicas de obtención de imagen. La búsqueda se organizó de acuerdo a las siguientes palabras clave y su respectiva traducción al inglés: protección gonadal, radiografía de pelvis, radiología pediátrica, dosis de radiación, radiosensibilidad. Los resultados se estructuraron para su lectura en cuatro subgrupos que son los siguientes: posicionamiento del protector gonadal, dosis de radiación, evolución de valores de radiosensibilidad y factores psicológicos.

\section{Resultados \\ Posición del protector gonadal}

En la publicación 121 de ICRP del año 2013(7) para protección radiológica en diagnóstico pediátrico y radiología intervencional, se plantea que una buena técnica radiográfica incluye el uso estándar de plomo o protección equivalente cerca de la zona a estudiar a modo de protección contra la radiación dispersa y extra focal. Estructuras tales como las gónadas deben protegerse si se encuentran dentro de los $5 \mathrm{~cm}$ del haz primario, siempre que sea posible sin perjudicar la información diagnóstica. Esto solo funciona cuando la protección está correctamente ubicada ya que, de estar mal ubicada, puede empeorar la calidad de la imagen y, en algunos casos, el blindaje no ser apropiado.

La ubicación de los protectores es diferenciado en mujeres y hombres (Figura 1); en mujeres este suele estar en la línea media sagital de la pelvis (pelvis verdadera); en hombres se ubica más bajo de la sínfisis púbica en la región del escroto ${ }^{(8)}$. Generalmente la ubicación ovárica en lactantes es más craneal, lo cual es más consistente con el desarrollo embriológico de las gónadas ${ }^{(9)}$. Esto último ha provocado un mal posicionamiento de los protectores gonadales documentado en algunos estudios ${ }^{(10,11)}$ ya sea por posición inadecuada, tamaño insuficiente 0 desplazamiento durante la adquisición de la imagen. Además, los ovarios pueden estar fuera de la pelvis

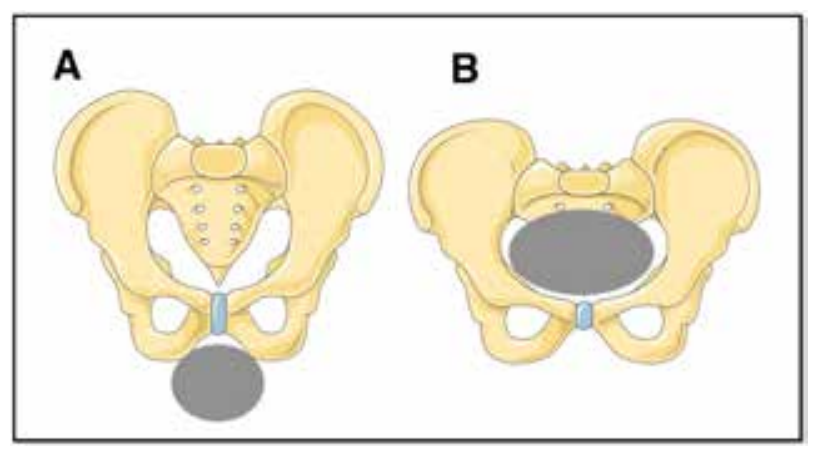

Figura 1: Ubicación ideal de protectores gonadales en pelvis masculina $(A)$ y femenina $(B)$. 
verdadera o cuenca pélvica en el $18 \%$ de la población y la posición del ovario puede variar dependiendo en el llenado de la vejiga(10).

Frantzen y col.(6) realizaron un estudio retrospectivo en 500 radiografías de pelvis en el que los protectores gonadales estaban colocados incorrectamente en el $91 \%$ de las radiografías de las niñas y se requirió una nueva imagen para el $28 \%$ de ellas.

Otro factor de importancia a considerar es que al día de hoy la mayoria de los equipos utilizados en radiodiagnóstico poseen un control de exposición automática (AEC). Su funcionamiento se basa en detectores cercanos al receptor de imagen que ajustan la salida de radiación mediante el monitoreo de kerma en aire en el receptor durante el disparo, el cual termina cuando la dosis de kerma-aire ha alcanzado su umbral con el fin de obtener una imagen óptima con la menor cantidad de radiación posible, evitando la subexposición o sobreexposición. Cuando un objeto radiopaco es ubicado entre la fuente del haz de radiación y el AEC provoca que menos rayos $X$ lleguen al detector, genera que el tubo de rayos emita más fotones, lo cual repercute en un aumento de la radiación a los órganos del cuerpo que no están cubiertos por el protector gonadal ${ }^{(12)}$. El consejo general establece que en radiografías de pelvis femeninas no deben usarse protectores gonadales junto con $A E C^{(13)}$. Como ya se ha señalado, la ubicación del protector gonadal femenino tiene un alto porcentaje de error en su ubicación lo que no solo induce a una mayor dosis en los ovarios sino que también a otros órganos más radiosensibles como es el caso del colon. Kaplan et al (2018) ${ }^{(14)}$ reportaron aumento de $63 \%$ y $147 \%$ en la dosis en fantomas de 5 años y adultos cuando los protectores eran usados en conjunto con AEC.

\section{Dosis de radiación}

La dosis de radiación involucrada actualmente en la radiografía de pelvis de adultos está en valores aproximados entre los 2 y 4 mGy ${ }^{(6,15)}$. En los exámenes pediátricos los valores son menores. En caso de múltiples países pertenecientes a la Unión Europea los valores de $\mathrm{DRL}$ en pelvis de niños está en valores menores a $1 \mathrm{mGy}{ }^{(16)}$. Una publicación chilena reporta un valor de 0,118 mGy en exámenes radiográficos de pelvis de 3-6 meses de edad(17).

Una comparación de estos valores a lo largo del tiempo muestra que las dosis de radiación han disminuido progresivamente en el tiempo. Kemerink y colaboradores ${ }^{(18)}$, investigando sobre el origen de la práctica del blindaje de gónadas, buscaron información sobre dosis de radiación de las radiografías de principios de siglo XX (año 1900). La búsqueda evidenció que tales datos no existen, probablemente porque la dosimetría, tal como se conoce hoy, y solo se introdujo alrededor de 1927. Esta publicación señala que durante el período de 1896-2018 la dosis de superficie de entrada de las radiografías pélvicas se redujo en un factor de aproximadamente 400.

Kotre ${ }^{(19)}$ utilizó una base de datos de exámenes radiográficos y fluoroscópicos en Reino Unido, incluyendo factores de la técnica radiográfica y sobre el equipo de rayos $X$, para estimar las dosis de radiación de pacientes y personal para los años 1899 a 1902. Las distribuciones de ESD (Dosis en superficie de entrada) para estos exámenes muestran valores de 189 mGy para los exámenes de abdomen / pelvis, 68 mGy para los exámenes de tórax y 354 mGy para los exámenes de tórax laterales. Estos pueden compararse con los valores medios actuales de ESD de $4.1 \mathrm{mGy}$ para abdomen anteroposterior (AP), 3.2 mGy para AP pelvis, 0.14 mGy para vistas AP de tórax y 0.51 mGy para tórax lateral. Esto otorga una proporción largamente superior de ESD (aproximadamente 52 veces mayor para los exámenes de abdomen y pelvis, 486 veces mayor para los exámenes de tórax y 694 veces mayor para los exámenes de tórax laterales).

En resumen, el blindaje de gónadas fue motivado inicialmente para prevenir la esterilidad masculina, pero se suspendió después de que las dosis disminuyeron, para luego ser retomado a mediados de la década de 1950, pero ahora con la finalidad de reducir los riesgos hereditarios. La evolución tecnológica y la optimización han sido capaces de reducir las dosis de gónadas al $0.5-2 \%$ de los valores que se generaban a principios del siglo pasado (aprox. 1905) ${ }^{(20)}$.

\section{Radiosensibilidad}

La Comisión Internacional de Protección Radiológica (ICRP) publica factores de ponderación (wt) destinados a la estimación del riesgo estocástico de los órganos expuestos a la radiación. En 1977, la Publicación 26 de la ICRP estimó por primera vez el peso de las gónadas en 0.25 , lo que suponía que las gónadas tenían un $25 \%$ del riesgo biológico durante un evento de radiación en todo el cuerpo ${ }^{(5)}$. La edición siguiente, la ICRP Publication 60 del año 1991, redujo esa estimación al $20 \%(\mathrm{Wt}=0.20)^{(21)} \mathrm{y}$, más recientemente, la Publicación 103 de ICRP del año 2007 redujo aún más las estimaciones del riesgo gonadal a un peso de $0.08^{(4)}$. Al mismo tiempo, esta última publicación aumentó las estimaciones de riesgo para el colon y el estómago. A estos órganos no se les había asignado peso individual en las primeras estimaciones de la ICRP, pero ahora se estima que tienen un peso de 0.12 cada uno, un riesgo combinado significativamente mayor que el de las gónadas. Este escenario cambiante de susceptibilidad a la radiación sugiere que la protección gonadal persiste hoy en día debido a preocupaciones sobre el riesgo de radiación fuera de lugar y que han ido quedando obsoletas ya que inicialmente la protección gonadal se introdujo como medida de disminución de riesgo hereditario (es decir, mutaciones en las células germinales que 
pueden afectar a las generaciones futuras), no estocástico ${ }^{(12)}$.

La Comisión afirma que no hay evidencia directa de que la exposición de los pacientes a la radiación provoque un exceso de enfermedades hereditarias en la descendencia. Sin embargo, y a pesar de no haber sido reportado riesgo genético a la descendencia en humanos hasta la fecha, la comisión considera que hay pruebas convincentes de tal efecto en animales de experimentación y, por lo tanto, sigue incluyendo el riesgo en su sistema de protección radiológica. Gran parte de los datos considerados para las recomendaciones de la ICRP de 2007 provienen de los informes revisados por el Comité Científico de las Naciones Unidas sobre los Efectos de la Radiación Atómica (UNSCEAR) 2001(22) en donde una de las publicaciones informó que las muertes por cáncer de ovario aumentaron con el aumento de las dosis de radiación los sobrevivientes de la bomba atómica(23). Sin embargo, el informe UNSCEAR 2001 argumenta que, sobre la base de datos genéticos de ratones y sobrevivientes de bombas atómicas, el riesgo hereditario ha sido ampliamente sobreestimado. La ICRP también reconoce que existen diferencias significativas en el riesgo según género y la edad de una persona al momento de la exposición. Sin embargo y a pesar de esto, la Comisión continúa recomendando que los coeficientes de riesgo nominales se apliquen a poblaciones enteras y no a individuos en la creencia de que esta política proporciona un sistema general de protección que es más simple y robusto. A pesar de la reducción en los factores de ponderación de los tejidos, la Comisión enfatizó que esto no proporciona justificación alguna para permitir que las exposiciones controladas de las gónadas aumenten en magnitud. Es necesario recalcar que los factores de ponderación tisulares son un promedio para edad y sexo, y representan valores medios para humanos. Por lo tanto, se supone que son válidos para ambos sexos y todos los grupos de edad. Al considerar a los niños pequeños como una subpoblación particularmente radiosensible, cualquier exposición innecesaria a la radiación a las gónadas puede considerarse inaceptable incluso en vista de las reducciones del valor del factor de ponderación tisular. Por lo tanto, la ICRP probablemente no aprobaría una medida para abandonar por completo el uso del blindaje gonadal, al menos en las niñas ${ }^{(24)}$.

Con respecto al tema de la protección de pacientes pediátricos, la ICRP tiene un reporte específico (ICRP 121) del año 2013 que señala, sin muchas recomendaciones de tipo práctico, que el blindaje gonadal puede ser imposible en muchos $\operatorname{casos}^{(7)}$. Las directrices para los tecnólogos médicos establecen que los protectores no deben colocarse en una posición que pueda interferir con la capacidad del software de autoexposición (AEC) ${ }^{(25)}$. Se requeriría que los tecnólogos apaguen el AEC para las radiografías de pelvis femenina antes de realizar un examen con blindaje. Esta excepción al flujo de trabajo normal eventualmente podría generar un error sistemático en la práctica ya propensa a errores de posicionamiento del blindaje gonadal ${ }^{(14)}$.

La ICRP en esta publicación (ICRP 121) es explícita en señalar que la protección de mamas, las gónadas y / o la tiroides, si se encuentran dentro de los $5 \mathrm{~cm}$ del haz primario, debe realizarse siempre que sea posible sin afectar la información de diagnóstico necesaria. Para exposiciones de 60-80 kV, se puede obtener una reducción máxima de la dosis gonadal de aproximadamente $30-40 \%$ protegiéndola con $0.25 \mathrm{~mm}$ de material equivalente de plomo inmediatamente en el borde del campo. Sin embargo, esto solo es cierto cuando la protección se coloca correctamente en el borde del campo. Si está mal colocado, dicho blindaje puede empeorar la calidad de la imagen, y en algunos casos, el blindaje puede no ser apropiado ${ }^{(7)}$.

\section{Factor psicológico}

Un aspecto destacable es la percepción que tienen los pacientes, los padres, y las personas en general respecto a la radiación ${ }^{(26)}$. Los pacientes 0 acompañantes están acostumbrados a la protección radiológica y no brindar el protector gonadal en estos procedimientos puede considerarse para ellos como una negligencia médica importante.

En muchos de los casos los pacientes se sienten seguros y se les provee tranquilidad al utilizar protector gonadal. Esto se debe a que se asocia la radiación con efectos negativos por lo cual los pacientes creen que al exponerse a la radiación en una radiografía de pelvis esta repercutirá negativamente en su organismo, sin embargo, la responsabilidad del personal encargado de los procedimientos es la de informar y dar tranquilidad a los pacientes respecto a este tema. Los pacientes también podrían confundir los riesgos estocásticos a niveles muy bajos de radiación, que son las utilizadas en exámenes pediátricos, con los riesgos deterministas a altos niveles de radiación que pueden resultar en infertilidad para pacientes. Las preocupaciones de los pacientes pueden ser atendidas si ellos saben que el tecnólogo médico encargado de su examen está prestando atención a su seguridad y que la falta de blindaje es intencional en lugar de negligente ${ }^{(27)}$.

\section{Discusión}

¿Es adecuado el uso de protector gonadal en pacientes pediátricos? Esta pregunta ha sido desarrollada teniendo en consideración la mayor cantidad de factores que influyen en su uso en el día de hoy de los cuales se consideran aspectos técnicos y psicológicos en la protección gonadal. Las radiografías pélvicas 
se realizan en la población infantil para diagnosticar patologías del anillo pélvico, así como lesiones en cadera. En nuestro país es destacable que el uso de radiografías pélvicas es parte de un protocolo de screening (cribado) para detectar precozmente en niños y niñas portadores de displasia de desarrollo de caderas (DDC) (28). Consideramos que la protección radiológica se debe basar en uno de los principios esenciales en radiodiagnóstico que es la optimización de las dosis, siendo esta la única herramienta disponible para el resguardo de la seguridad del paciente ${ }^{(4)}$. La optimización se logra manteniendo las dosis tan bajas como sean razonablemente posibles con el fin de obtener la calidad de imagen requerida e información diagnóstica deseada. Los protectores gonadales tienen una eficacia cuestionable y dados los nuevos desarrollos en la formación de la imagen en radiodiagnóstico, los mecanismos de seguridad y control de dosis, su uso puede ser innecesario, siempre teniendo en consideración factores de optimización de dosis, los cuales pueden ser logrados determinado los niveles de dosis impartidos a los pacientes y establecer niveles referenciales para el diagnóstico, que son el rango de valores de dosis aceptable para un paciente u objeto antropomorfo ${ }^{(4)}$. En esta línea, un trabajo pionero en Chile fue realizado por Nocetti et al (2014) ${ }^{(17)}$ en los que mide los niveles de dosis en lactantes en la radiografía de pelvis, replicable en la determinación del nivel referencial diagnóstico en niños con edades entre 3 a 6 meses. Nuestro país no obliga a estimar las dosis impartidas a los pacientes en los procedimientos diagnósticos con radiaciones ionizantes. Lo que quizás sea aún más preocupante es que tampoco define criterios mínimos de calidad de imagen en radiodiagnóstico ${ }^{(29)}$ lo cual es muy diferente a lo que ocurre en la comunidad internacional, donde se han establecido protocolos en común con el fin de regular dosis y criterios de calidad en imágenes pediátricas de radiodiagnóstico ${ }^{(30)}$. Consideramos que una mayor sensibilización de los aspectos técnicos, tanto del personal que se desempeña en la práctica radiológica, así como de las autoridades, nos llevarán a un mayor control y mejora en las prácticas de radiodiagnóstico pediátrico.

Desde la incorporación de los protectores gonadales en la década de los 50 hasta la fecha, ha existido un drástica mejora en los sistemas de adquisición de imágenes ${ }^{(31)}$, los cuales consideran una mejor colimación, exámenes con menores tiempos de exposición, los que han ampliado el uso de exposímetros automáticos en pacientes pediátricos, la incorporación de radiología digital, la cual posee un amplio rango dinámico, y un post-procesamiento de las imágenes digitales reduciendo la cantidad de radiación para producir una imagen de valor diagnóstico.

Hoy, después de la optimización, la reducción del riesgo hereditario se considera muy marginal.
La evaluación de las dosis pélvicas reveló una gran reducción en los riesgos de radiación facilitada por los desarrollos tecnológicos. La optimización también contribuyó, pero desafortunadamente, su potencial nunca ha sido explotado completa y adecuadamente. Hoy, usando un equipo de rayos $X$ moderno y optimizado, el blindaje de gónadas puede ser descontinuado de manera segura para las mujeres. Para los hombres, puede haber un beneficio marginal, pero los posibles efectos secundarios negativos hacen cuestionar su uso. La interrupción de la protección de las gónadas, por lo tanto, parece justificable en el momento actual(20). Esto es algo que la profesión radiológica debería tomar en serio dada la orientación y la legislación basados en datos de larga data. EI principio "Tan bajo como sea razonablemente posible" (ALARA) se remonta a 1966 y el requisito para optimizar es de 1973.

\section{Conclusión}

El beneficio de la protección de las gónadas en la radiografía pélvica anteroposterior (AP) está actualmente en debate. Mientras algunos siguen respaldando la medida, otros organismos y autores ya no la respaldan y descartan el blindaje de gónadas en parte o expresan dudas sobre los beneficios.

Es necesario reconsiderar las prácticas actuales que, como se hemos visto, están fundadas en consensos científicos que ya han sido reemplazados por nuevos conocimientos y avances técnicos, y considerar el factor de cambio cultural basados en estos nuevos consensos para su implementación, sin generar mayor preocupación en nuestros pacientes.

\section{Referencias}

1. Adran GM. Protection of the male gonads in diagnostic procedures. Br J Radiol. 1957; 353: 280.

2. Abram E, Wilkinson DM, Hodson CJ. Gonadal protection from X radiation for the female. Br J Radiol 1958; 366: 335-336.

3. Whitehead G, Griffiths JT. The leicester gonad protector: A device to afford localised. Br J Radiol 1961; 135-136.

4. International Commission on Radiological Protection (ICRP). Las Recomendaciones 2007 de la Comisión Internacional de Protección Radiológica. Publicación 103. 2007.

5. International Commission on Radiological Protection (ICRP). Recommendations of the Radiological Protection. Publication 26. 1977.

6. Frantzen MJ, Robben S, Postma AA, Zoetelief J, Wildberger JE, Kemerink GJ. Gonad shielding in paediatric pelvic radiography: Disadvantages prevail over benefit. Insights Imaging. 2012; 3(1): 23-32.

7. Khong PL, Ringertz H, Donoghue V, Frush D, Rehani $\mathrm{M}$, Appelgate $\mathrm{K}$, et al. Radiological protection in paediatric diagnostic and interventional radiology. Ann ICRP. 2013; 42(2): 11.

8. Bardo DME, Black M, Schenk K, Zaritzky MF. Location of the ovaries in girls from newborn to 18 years of age: 
Reconsidering ovarian shielding. Pediatr Radiol. 2009; 39(3): 253-259.

9. Sadler T.W. Langman's medical embryology. Urogenital System. 14th edition. Philadelphia: Wolters Kluwer. 2019; pp 260-272.

10. Fawcett SL, Gomez AC, Barter SJ, Ditchfield M, Set P. More harm than good? The anatomy of misguided shielding of the ovaries. Br J Radiol. 2012; 85(1016): 442-447.

11. Lee MC, Lloyd J, Solomito MJ. Poor utility of gonadal shielding for pediatric pelvic radiographs. Orthopedics. 2017; 40(4): e623-e627.

12. Kaplan SL, Magill D, Felice MA, Edgar JC, Anupindi SA, Zhu X. Intussusception reduction: Effect of air vs. liquid enema on radiation dose. Pediatr Radiol. 2017; 47(11): 1471-1476.

13. Goske MJ, Charkot E, Herrmann T, John SD, Mills TT, Morrison G, et al. Image Gently: Challenges for radiologic technologists when performing digital radiography in children. Pediatr Radiol. 2011; 41(5): 611-619.

14. Kaplan SL, Magill D, Felice MA, Xiao R, Ali S, Zhu X. Female gonadal shielding with automatic exposure control increases radiation risks. Pediatr Radiol. 2018; 48(2): 227-234.

15. Hart D., Hillier MC., Shrimpton PC. Doses to Patients from Radiographic and Fluoroscopic X-ray Imaging Procedures in the UK-2010 Review Report. HPACRCE-034. Health Protection Agency, Chilton. 2012: Disponible en: https://www.gov.uk/government/ publications/diagnostic-radiology-national-diagnosticreference-levels-ndrls (Accesado el 16/mar/2020).

16. Bosmans H., Damilakis J., Ducou le Pointe H., Foley SJ. Radiation Protection N ${ }^{\circ} 185$ European Guidelines on Diagnostic Reference Levels for Paediatric Imaging. UCD. 2008. http://hdl.handle.net/10197/10396

17. Nocetti D., Leyton F. Niveles de dosis efectiva a lactantes en radiografía de pelvis. Primeros datos para Chile. Interciencia 2014; 39(7): 502-505.

18. Kemerink GJ., et al. The skin dose of pelvic radiographs since 1896. Insights into imaging. 2019; 10(1): 39.

19. Kotre CJ., Little BG. Patient and staff radiation doses from early radiological examinations (1899-1902). The British Journal of Radiology. 2006; 79(946): 837-842. doi:10.1259/bjr/16982267

20. Jeukens C, et al. Gonad shielding in pelvic radiogra- phy: modern optimised X-ray systems might allow its discontinuation. Insights into Imaging. 2020; 11(1): 15.

21. International Commission on Radiological Protection (ICRP). Publication 60. 1990 Recommendations of the International Commission on Radiological Protection. Elsevier Health Sciences. 1991.

22. United Nations Scientific Committee on the Effects of Atomic Radiation (UNSCEAR). Heritable Effects of Radiation, 2001 Report to the General Assembly with Scientific Annex. New York, NY: United Nations, 2001.

23. Pierce DA, Shimizu Y, Preston DL, Vaeth M, Mabuchi $\mathrm{K}$. Studies of the mortality of atomic bomb survivors. Report 12, part 1. Cancer: 1950-1980. Radiation Research 1996; 146: 1-27.

24. Fawcett SL., Barter SJ. The use of gonad shielding in paediatric hip and pelvis radiographs. The British Journal of Radiology. 2009; 82(977): 363-370.

25. Herrmann TL, Fauber TL, Gill J, et al. White paper: best practices in digital radiography. American Society of Radiologic Technologists 2012. https://www.asrt. org/docs/default-source/publications/whitepapers/ asrt12_bstpracdigradwhp_final.pdf. Accessed 15 Mar 2020.

26. Freudenberg LS, Beyer T. Subjective perception of radiation risk. J Nucl Med. 2011; 52(Suppl 2): 29-36.

27. Marsh RM, Rm M, Silosky M. Patient Shielding in Diagnostic Legacy Practice. Am J Roentgenol. 2019; 212(1): 1-3.

28. Ministerio de salud. Regula examen de medicina preventiva. Exenta ${ }^{\circ} 1.236$ Chile. 2009; p.1-23. Available from: https://www.minsal.cl/portal/url/item/9556d35ef 058000ce04001011f016d71.pdf

29. Ministerio de Salud. Reglamento sobre autorizaciones para instalaciones radiactivas o equipos generadores de radiaciones ionizantes, personal que se desempeña en ellas, $u$ opere tales equipos y otras actividades afines. Chile; 1984; 17-20.

30. European Commission. European Guidelines on Quality Criteria for Diagnostic Radiographic Images. Eur 16260 En 1996: 71 p.

31. International Atomic Energy Agency (IAEA). Radiation protection in Paediatric Radiology, Safety reports series $\mathrm{n}^{\circ} 71$, IAEA, Vienna. IAEA Saf Reports Ser. 2012; 71: 128. 